\title{
Genetic mechanisms underlying yield potential in the rice high-yielding cultivar Takanari, based on reciprocal chromosome segment substitution lines
}

\author{
Toshiyuki Takai ${ }^{1,2}$, Takashi Ikka², Katsuhiko Kondo², Yasunori Nonoue³, Nozomi Ono ${ }^{3}$, Yumiko Arai-Sanoh', \\ Satoshi Yoshinaga ${ }^{1}$, Hiroshi Nakano ${ }^{1}$, Masahiro Yano ${ }^{2}$, Motohiko Kondo ${ }^{1}$ and Toshio Yamamoto ${ }^{2 *}$
}

\begin{abstract}
Background: Increasing rice yield potential is a major objective in rice breeding programs, given the need for meeting the demands of population growth, especially in Asia. Genetic analysis using genomic information and high-yielding cultivars can facilitate understanding of the genetic mechanisms underlying rice yield potential. Chromosome segment substitution lines (CSSLS) are a powerful tool for the detection and precise mapping of quantitative trait loci (QTLs) that have both large and small effects. In addition, reciprocal CSSLS developed in both parental cultivar backgrounds may be appropriate for evaluating gene activity, as a single factor or in epistatic interactions.

Results: We developed reciprocal CSSLs derived from a cross between Takanari (one of the most productive indica cultivars) and a leading japonica cultivar, Koshihikari; both the cultivars were developed in Japan. Forty-one CSSLs covered most of the Takanari genome in the Koshihikari background and 39 CSSLs covered the Koshihikari genome in the Takanari background. Using the reciprocal CSSLS, we conducted yield trials under canopy conditions in paddy fields. While no CSSLs significantly exceeded the recurrent parent cultivar in yield, genetic analysis detected 48 and 47 QTLS for yield and its components in the Koshihikari and Takanari backgrounds, respectively. A number of QTLs showed a trade-off, in which the allele with increased sink-size traits (spikelet number per panicle or per square meter) was associated with decreased ripening percentage or 1000-grain weight. These results indicate that increased sink size is not sufficient to increase rice yield in both backgrounds. In addition, most QTLs were detected in either one of the two genetic backgrounds, suggesting that these loci may be under epistatic control with other gene(s).

Conclusions: We demonstrated that the reciprocal CSSLs are a useful tool for understanding the genetic mechanisms underlying yield potential in the high-yielding rice cultivar Takanari. Our results suggest that sink-size QTLs in combination with QTLs for source strength or translocation capacity, as well as careful attention to epistatic interactions, are necessary for increasing rice yield. Thus, our findings provide a foundation for developing rice cultivars with higher yield potential in future breeding programs.
\end{abstract}

Keywords: Chromosome segment substitution lines (CSSLs), Quantitative trait locus (QTL), Rice, Yield potential

\section{Background}

Increasing crop productivity is a global challenge and is necessary for keeping pace with population growth worldwide [1]. More than half of the world's population is in Asia, where rice is grown and consumed as a staple food [2]. The predicted population growth in Asia will require a $60-70 \%$ increase in rice production by 2050 ,

\footnotetext{
* Correspondence: yamamo101040@affrc.go.jp

${ }^{2}$ National Institute of Agrobiological Sciences, Tsukuba, Ibaraki 305-8602, Japan Full list of author information is available at the end of the article
}

but there is insufficient space for a corresponding increase in agriculture [3]. To meet the anticipated demand, it is necessary to increase rice production by improving potential rice yield per unit land area.

In the tropics, the yield potential of current highyielding inbred rice cultivars is $10 \mathrm{t} \cdot \mathrm{ha}^{-1}$ as unhulled rice under favorable irrigated conditions [4]. This yield potential was first attained by IR8, the first modern high-yielding cultivar released by the International Rice Research Institute (IRRI) in the late 1960s. The release of IR8 and subsequent 
high-yielding cultivars helped to more than double rice production over the past half century. This successful increase in production was called the "Green Revolution" in rice [5]. However, recent trends in yield in tropical environments indicate that yield potential has stagnated since the release of IR8 [6].

In temperate Japan, high-yielding rice has been developed using the indica and japonica cultivars since the 1980s [7]. The latest yield trials, conducted using recently developed high-yielding cultivars, produced nearly $10 \mathrm{t} \cdot \mathrm{ha}^{-1}$ as brown rice $\left(>12 \mathrm{t} \cdot \mathrm{ha}^{-1}\right.$ as unhulled rice yield) in eastern Japan [8] and $>10 \mathrm{t} \cdot \mathrm{ha}^{-1}$ as brown rice in western Japan [9]. Among the individual trials, a brown rice yield of $11.7 \mathrm{t} \cdot \mathrm{ha}^{-1}$ was reported in western Japan [9]. To our knowledge, this represents the highest yield recorded in Japan to date, and was attained using Takanari, a high-yielding indica cultivar. Takanari is a semidwarf cultivar descended from high-yielding cultivars including IR8 [10]. Ecophysiological studies have characterized Takanari as having large sink size as a result of high spikelet number per panicle, strong source characteristics (e.g., high photosynthesis rate), and high carbohydrate translocation capacity [11-14]. Therefore, it is important to understand the genetic mechanisms underlying the high yield potential in Takanari to further improve this potential.

Over the past two decades, advances in molecular genetics technology using the complete rice genome sequence have facilitated genetic analyses, including the mapping and cloning of quantitative trait loci (QTLs) that control complex traits $[15,16]$. Chromosome segment substitution lines (CSSLs), which carry a specific donor chromosome segment in the genetic background of a recurrent cultivar, are powerful tools for enhancing the potential of genetic analysis. CSSLs are appropriate for detecting QTLs with both large and small effects that are often masked by other QTLs with large effects in primary populations, such as $\mathrm{F}_{2}$ populations and recombinant inbred lines $[17,18]$. Because yield is a highly complex trait that is controlled by a large number of QTLs with small effects, CSSLs are useful for understanding the genetic mechanisms underlying this characteristic. To date, several CSSLs have been developed in rice for several cross combinations [17,19-23], including reciprocal CSSLs [20,21]. Reciprocal CSSLs have the advantage of enabling evaluation of differences in allelic effects of QTLs in both genetic backgrounds. However, to our knowledge, genetic analysis of rice yield potential has not been conducted using reciprocal CSSLs. Therefore, the development of reciprocal CSSLs for yield trials using Takanari represents a promising approach.

In this study, we developed reciprocal CSSLs from a cross between Takanari and Koshihikari, a leading japonica cultivar, by repeated backcrossing, self-pollinating, and marker-assisted selection (MAS). The CSSLs in the Koshihikari background consisted of 41 lines covering the entire Takanari genome, and these are promising materials for detecting QTLs underlying high yield potential in Takanari. The CSSLs in the Takanari background consisted of 39 lines covering the entire Koshihikari genome, and they may enable detection of QTLs for increasing yield potential in Takanari. Yield trials using the reciprocal CSSLs revealed a number of QTLs associated with yield and its components in both genetic backgrounds. Our findings provide a foundation for developing rice cultivars with higher yield potential in future breeding programs.

\section{Methods}

\section{Development of the CSSLs}

Two rice cultivars, Takanari and Koshihikari, developed in Japan (Figure 1), were used to develop the reciprocal CSSLs using the procedure summarized in Figure 2. We conducted repeated reciprocal backcrossing and performed foreground (but not background) selection for the target chromosome segments until the $\mathrm{BC}_{3} \mathrm{~F}_{1}$ generation. From the $\mathrm{BC}_{4} \mathrm{~F}_{1}$ populations, all heterozygous regions were surveyed, and foreground and background selection were combined to select CSSLs. PCR-based DNA markers ( $n=141)$, including the previously developed gene markers GN1a, sd1, and APO1 [10,16,19,24-26], were used for MAS.

To develop CSSLs in the Koshihikari genetic background, the $F_{1}$ plants derived from a cross between Koshihikari and Takanari were backcrossed to Koshihikari to produce $95 \mathrm{BC}_{1} \mathrm{~F}_{1}$ plants. Then, we used MAS to select $23 \mathrm{BC}_{1} \mathrm{~F}_{1}$ plants carrying one or two target chromosome segments, based on the genotypes of 86 DNA markers distributed across the genome. These $23 \mathrm{BC}_{1} \mathrm{~F}_{1}$ plants were again backcrossed to Koshihikari to produce $\mathrm{BC}_{2} \mathrm{~F}_{1}$ seeds. We subsequently grew $408 \mathrm{BC}_{2} \mathrm{~F}_{1}$ individuals derived from the $23 \mathrm{BC}_{1} \mathrm{~F}_{1}$ plants, and selected $24 \mathrm{BC}_{2} \mathrm{~F}_{1}$ plants, carrying one or two heterozygous target segments, by MAS for

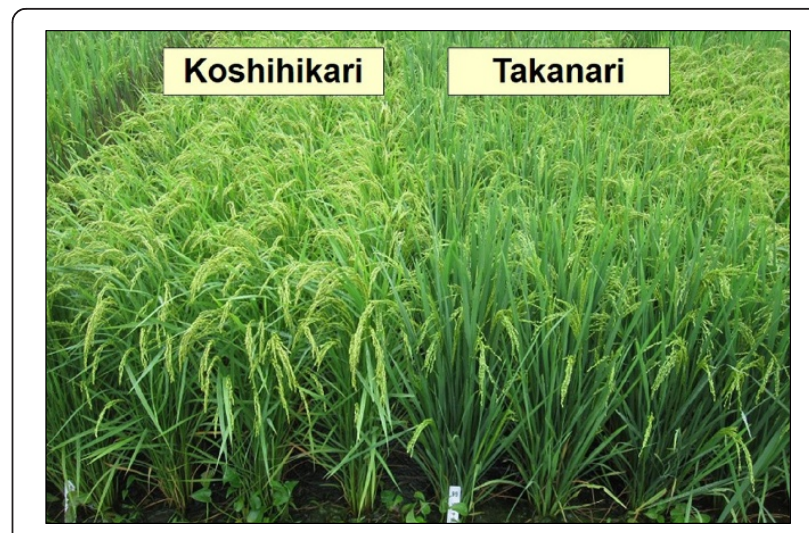

Figure 1 Image of Koshihikari and Takanari plants. 


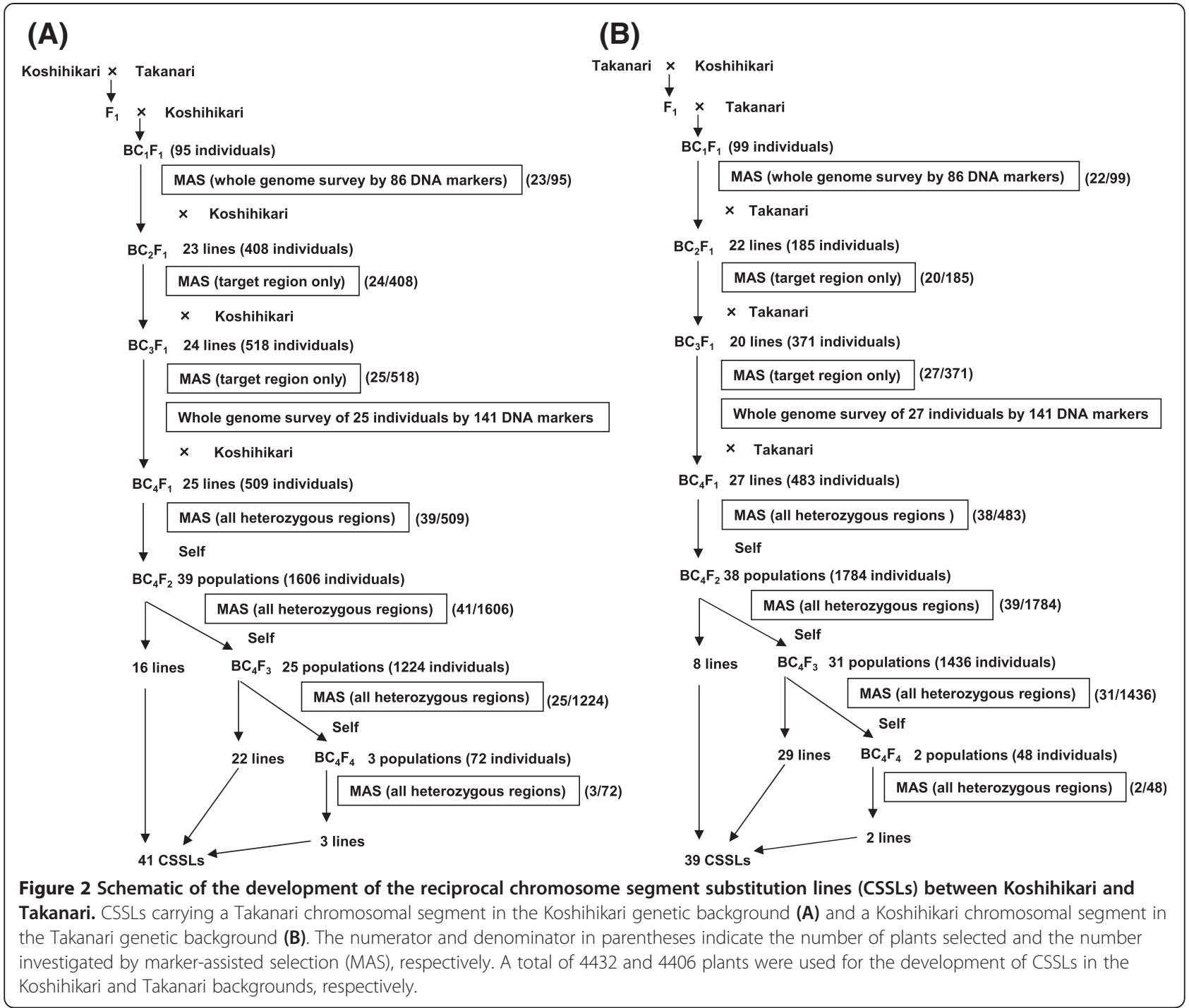

the subsequent backcross to Koshihikari to produce $\mathrm{BC}_{3} \mathrm{~F}_{1}$ seeds. In the same way, 25 out of $518 \mathrm{BC}_{3} \mathrm{~F}_{1}$ individuals derived from the $24 \mathrm{BC}_{2} \mathrm{~F}_{1}$ plants were selected by MAS for subsequent backcross to Koshihikari to produce $\mathrm{BC}_{4} \mathrm{~F}_{1}$ seeds. We surveyed the genotypes of the $25 \mathrm{BC}_{3} \mathrm{~F}_{1}$ plants by 141 genome-wide DNA markers for the subsequent target and background selection. Then, 39 out of $509 \mathrm{BC}_{4} \mathrm{~F}_{1}$ individuals derived from the $25 \mathrm{BC}_{3} \mathrm{~F}_{1}$ plants were selected by MAS for all heterozygous regions, including target segments. To obtain candidate plants as CSSLs homozygous for Takanari for the target segments, the $39 \mathrm{BC}_{4} \mathrm{~F}_{1}$ plants were self-pollinated, and the resulting $1606 \mathrm{BC}_{4} \mathrm{~F}_{2}$ individuals were surveyed by MAS to select $41 \mathrm{BC}_{4} \mathrm{~F}_{2}$ plants. Heterozygous segments for the non-target background remained in the $25 \mathrm{BC}_{4} \mathrm{~F}_{2}$ plants, so additional selfpollination and MAS were conducted to minimize the proportion of heterozygous regions in the background. Finally, 41 plants were selected as CSSLs (Figure 2A).
The CSSLs in the Takanari genetic background were developed using the same method as used for the Koshihikari background (Figure 2B). Finally, 39 plants were selected as CSSLs. Seeds of the reciprocal CSSLs can be obtained from the Rice Genome Resource Center (http://www.rgrc.dna. affrc.go.jp/index.html).

\section{Yield trials}

Yield trials were conducted in the experimental paddy field at the NARO Institute of Crop Science, Tsukubamirai $\left(36^{\circ} 02^{\prime} \mathrm{N}, 140^{\circ} 04^{\prime} \mathrm{E}\right)$, Ibaraki, Japan, in 2011 and 2012 . The soils were Gleyic Fluvisols. Reciprocal CSSLs (41 in the Koshihikari background and 39 in the Takanari background) and parent cultivars (Koshihikari and Takanari) were cultivated under irrigated conditions. Two paddy fields were prepared and each reciprocal CSSL was grown in each paddy field. Seeds were sown in a seedling nursery box on April 26, 2011, and April 25, 2012, and were 
transplanted (one seedling per hill) on May 19, 2011, and May 17, 2012, respectively. The planting density was 22.2 hills $\mathrm{m}^{-2}$, with $15 \mathrm{~cm}$ between hills and $30 \mathrm{~cm}$ between rows. The experimental plots $\left(5.7 \mathrm{~m}^{2}\right.$ each) were arranged in a randomized complete block design with three replications. Basal fertilizer was applied at a rate of $6 \mathrm{~g} \mathrm{~N} \mathrm{~m}^{-2}$ as controlled release fertilizer ( $2 \mathrm{~g}$ LP40, $2 \mathrm{~g}$ LPs100, and $2 \mathrm{~g}$ LP140), $5.2 \mathrm{~g} \mathrm{P} \mathrm{m}^{-2}$, and $7.5 \mathrm{~g} \mathrm{~K} \mathrm{~m}^{-2}$. LP40 and LP140 release $80 \%$ of their total nitrogen content at a uniform rate up to 40 and 140 days after application, respectively, at $20-30^{\circ} \mathrm{C}$. LPs 100 releases $80 \%$ of its total nitrogen content at a sigmoid rate up to 100 days after application at $20-30^{\circ} \mathrm{C}$.

Days-to-heading was defined as the number of days from sowing to heading of the first panicle in five plants for each CSSL and parent cultivar. At maturity, in midto late September, plants covering $1.8 \mathrm{~m}^{2}$ (40 hills) were harvested from each plot for determination of yield and its components. Panicle number was counted and the panicles were threshed to obtain unhulled grains, which were weighed and divided equally into subsamples A and B. Approximately $40 \mathrm{~g}$ of unhulled grains (subsample C) was selected from subsample A and counted using an electronic seed counter (KC-10S, Fujiwara Scientific Co. Ltd., Tokyo, Japan). Spikelet number per unit area $\left(\mathrm{m}^{2}\right)$ was calculated as the grain number in subsample $C$ divided by the weight of subsample $C$ and multiplied by the total weight of the unhulled grains per unit area. Spikelet number per panicle was calculated as the spikelet number per unit area divided by panicle number per unit area. The hulls from subsample B were subsequently removed with a rice huller $(25 \mathrm{M}$, Ohya Tanzo G.K. Company, Aichi, Japan), and the hulled grains were weighed to determine brown rice yield. The grains were then screened using a grain sorter (TWS, Satake Co. Ltd., Tokyo, Japan) with $1.6 \mathrm{~mm}$ sieve size and 1000-grain weight was calculated. Ripening percentage was calculated from the number of screened hulled grains divided by the spikelet number per unit area. Brown rice yield and 1000-grain weight were adjusted to $15 \%$ moisture content. Culm length was measured for five plants in each CSSL and parent cultivar at maturity.

\section{Statistical and genetic analyses}

Statistical analyses were performed using a general linear model with SPSS 22.0 (IBM, Chicago, IL). CSSL was considered as a fixed effect, and year and replication were considered as random effects. Analysis of variance (ANOVA) was conducted to examine the effects of CSSL on yield and its components. Based on the ANOVA results, significant CSSL effects $(P<0.05)$ were explored using Dunnett's test for yield and its components. In the Dunnett's test, Koshihikari was used as a control in the Koshihikari genetic background and Takanari was used as a control in the Takanari genetic background. To delineate candidate QTL regions, substitution mapping was conducted by comparing overlapping segments among the CSSLs according to our previous study [17].

\section{Results}

\section{Genotypes of the reciprocal CSSLs}

Graphical genotypes of 41 CSSLs in the Koshihikari background and 39 CSSLs in the Takanari background were determined using 141 DNA markers distributed evenly across the 12 rice chromosomes (Figure 3, Additional file 1: Figure S1). Each chromosome was covered by two to five lines carrying overlapping segments, except for a small region between DNA markers RM2935 and RM7344 on chromosome 12 in the Koshihikari background, which was not covered because of sterility when we selected a line carrying the segment homozygous for Takanari. Most CSSLs carried only one chromosome segment. However, a small segment was substituted in the genetic backgrounds in SL1240 and SL1315. SL1321 also carried two heterozygous segments and one homozygous segment for Koshihikari. The substituted segment size in each CSSL ranged from 6.9 $\mathrm{Mb}$ to $26.2 \mathrm{Mb}$ in the Koshihikari background and from 7.4 $\mathrm{Mb}$ to $27.1 \mathrm{Mb}$ in the Takanari background.

\section{Climate conditions and variation in days-to-heading in the reciprocal CSSLs}

Mean temperatures during the experimental period were similar in 2011 and 2012, and showed a gradual increase as the season progressed, until mid-September (Figure 4A). Solar radiation was relatively higher in late May and late August in 2012 compared with 2011 (Figure 4B). Koshihikari headed at approximately 99 days after sowing, and Takanari headed at 102-103 days after sowing (Additional file 2: Figure S2). In the Koshihikari background, SL1222 and SL1208 headed approximately 11 days earlier and 11 days later than Koshihikari, respectively. Earlier heading in SL1222 may be derived from the effect of $H d 1$, because the substituted segment in SL1222 contained Hd1 [28]. Late heading in SL1208 is discussed later. In the remaining 39 CSSLs, days-toheading ranged from 95 to 105 (within 6 days of Koshihikari). In the Takanari background, SL1320 and SL1323 did not head under the experimental conditions. No heading in SL1320 may be caused by the effect of $H d 1$, because the substituted segment in SL1320 contained Hd1 [28]. No heading in SL1323 may be caused by a new QTL because no QTL has been reported in the substituted region on the short arm of chromosome 7. In addition, SL1335 and SL1336 headed 17 and 29 days later than Takanari, respectively. Late heading in SL1335 and SL1336 is discussed later. On the other hand, the remaining 35 CSSLs headed at 97-108 days after sowing, which was 


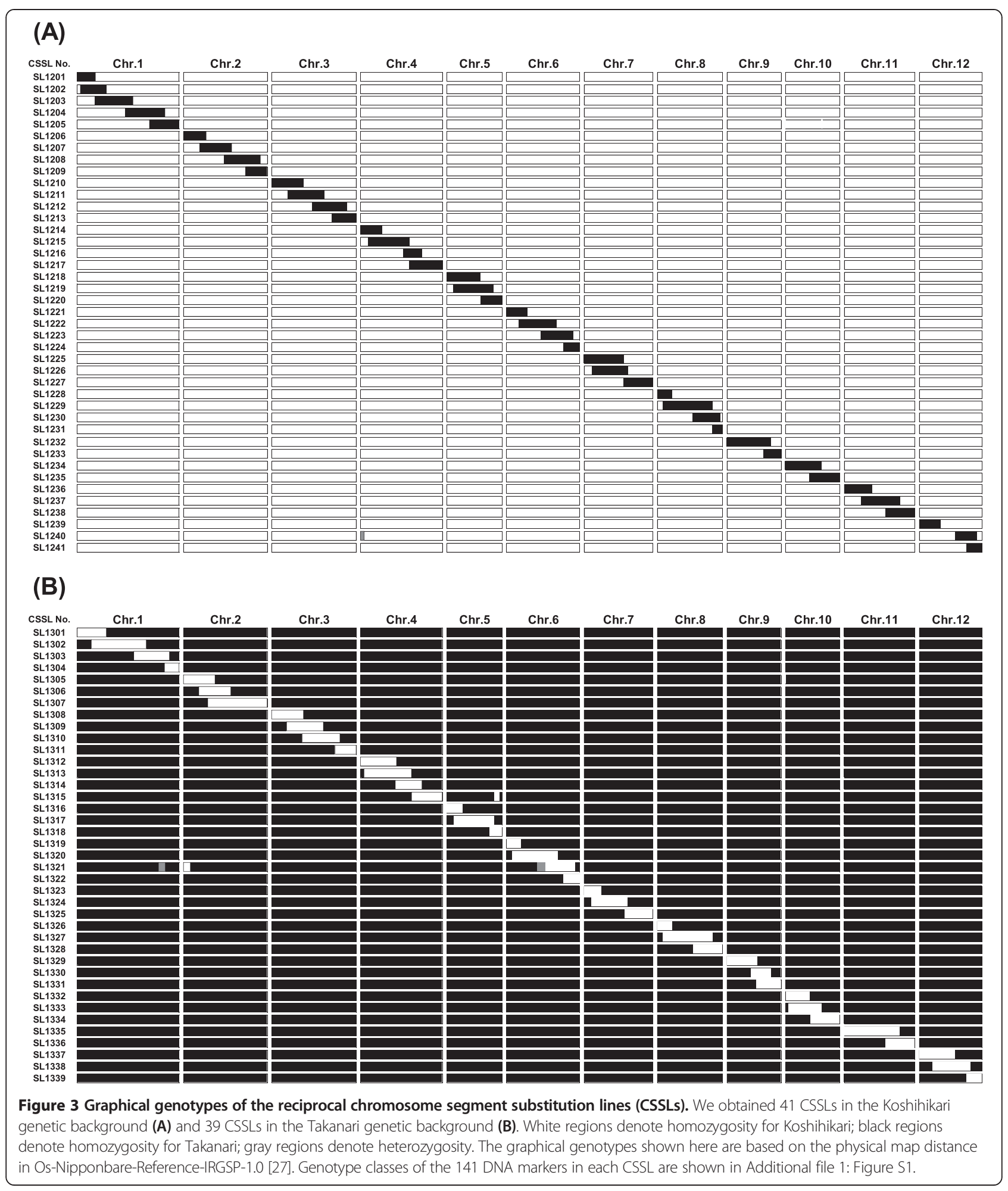

also within 6 days of Takanari. Therefore, we considered that most CSSLs and parent cultivars were grown under similar climate conditions.
Yield and its components in the reciprocal CSSLs

Takanari produced approximately $40 \%$ more spikelets per unit area than Koshihikari, which was a result of Takanari 


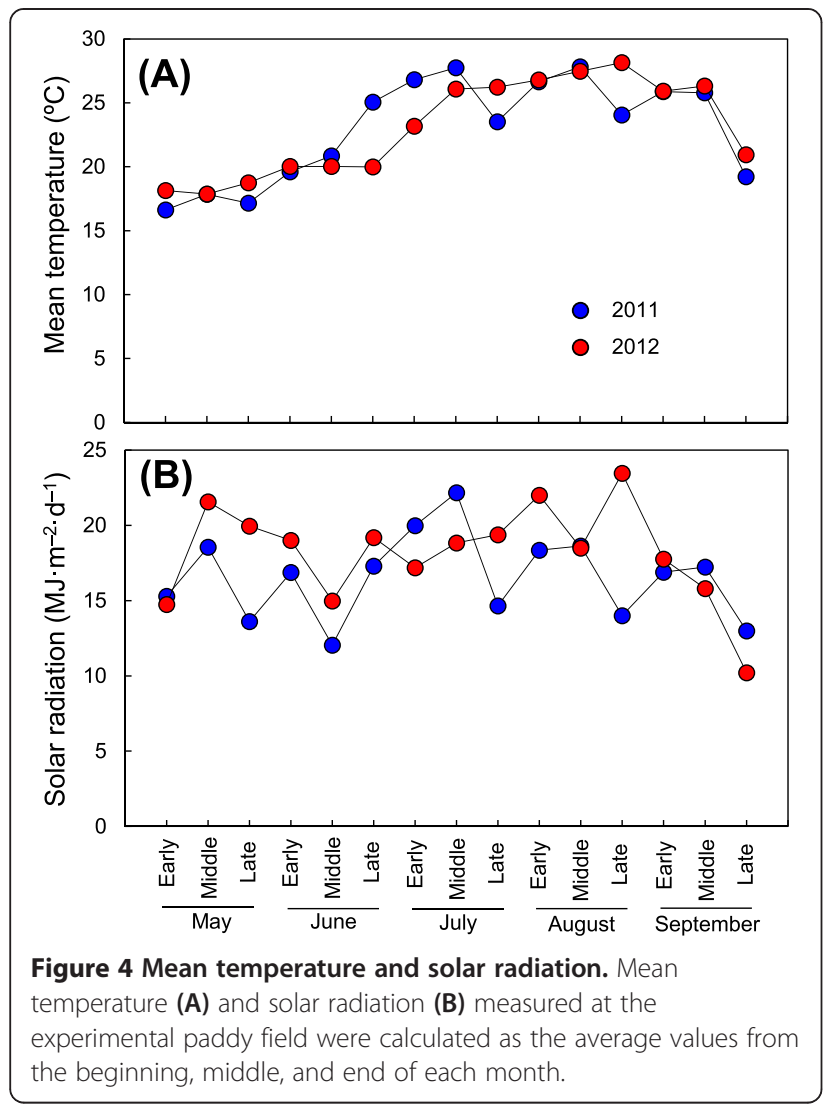

having $30 \%$ fewer panicles but twice as many spikelets per panicle (Figure 5). The same ripening percentage was obtained in Takanari and Koshihikari, although 1000-grain weight was 7\% lower in Takanari. Finally, brown rice yield was $27 \%$ higher in Takanari than in Koshihikari.

Previous studies identified and cloned QTLs for sinksize traits (spikelet number per panicle), including GN1a [29] and APO1 [30], and a semidwarf gene, $s d 1$ [31], as a single gene. Because we found sequence differences between Takanari and Koshihikari in these genes (Additional file 3: Figure S3), we first focused on the effects of the genes. SL1201 and SL1202, which carried the Takanari allele of GN1a in the Koshihikari background, produced $33 \%$ and $38 \%$ more spikelets per panicle and 22\% and 23\% more spikelets per square meter than Koshihikari, respectively (Figure 5A). However, these two CSSLs reduced ripening percentage and 1000-grain weight, and there was no difference in final brown rice yield between these CSSLs and Koshihikari. Meanwhile, SL1301, carrying the Koshihikari allele of GN1a in the Takanari background, produced $26 \%$ fewer spikelets per panicle and $20 \%$ fewer spikelets per square meter than Takanari (Figure 5B). Ripening percentage and 1000-grain weight in SL1301 were the same as in Takanari, and the final brown rice yield in SL1301 was 22\% lower than that in Takanari. Similar reciprocal effects of APO1 were observed for spikelet number per panicle; SL1223 and SL1224, which contained the Takanari allele of APO1, produced $19 \%$ and $13 \%$ more spikelets per panicle, respectively, whereas SL1321 and SL1322 (containing the Koshihikari allele of APO1) produced 17\% and 12\% fewer spikelets per panicle, respectively. However, the effects of APO1 did not lead to changes in final brown rice yield. Reciprocal effects were confirmed for culm length on the $s d 1$ gene; SL1205 (carrying the Takanari allele of $s d 1$ ) had shortened culms compared with Koshihikari, whereas SL1303 and SL1304 (carrying the Koshihikari allele of $s d 1$ ) had elongated culms compared with Takanari (Additional file 2: Figure S2). However, no effects of $s d 1$ were observed for brown rice yield and its components in the reciprocal backgrounds.

In addition to the CSSLs carrying GN1a, APO1, and $s d 1$, there were significant differences in brown rice yield and its components between Koshihikari and some CSSLs in the Koshihikari genetic background (Figure 5A), and between Takanari and some CSSLs in the Takanari genetic background (Figure 5B). Although some CSSLs had positive values for a yield component, they did not produce significantly higher yield than the recurrent parental cultivar in both backgrounds. For example, SL1310 (Takanari background) produced 19\% more panicles and 20\% more spikelets per square meter than Takanari, but had reduced ripening percentage and 1000-grain weight. Therefore, the final brown rice yield in this CSSL was similar to that in Takanari.

\section{QTL mapping for yield and its components}

We detected 48 and 47 QTLs for yield and its components in the Koshihikari and Takanari backgrounds, respectively (Figure 6).

In the Koshihikari background, three QTLs for panicle number were identified, one of which increased and two of which decreased panicle number in plants with the Takanari allele (Figure 6A). Twelve QTLs for number of spikelets per panicle were detected, seven with positive effects and five with negative effects on spikelet number in plants with the Takanari allele. Considering the effects of the QTLs and the chromosomal regions, the loci on the short arm of chromosome 1 and on the long arm of chromosome 6 were regarded as GN1a and APO1, respectively. Six QTLs were found for spikelet number per square meter; half of these increased and half decreased the spikelet number in plants with the Takanari allele. The five QTLs identified for ripening percentage all had negative effects on plants with the Takanari allele. Sixteen QTLs were detected for 1000-grain weight; of these, seven increased and nine reduced the value of this yield component in plants with the Takanari allele. Six QTLs were identified for brown rice yield, and all had negative effects on plants with the Takanari allele (Figure 6A). 


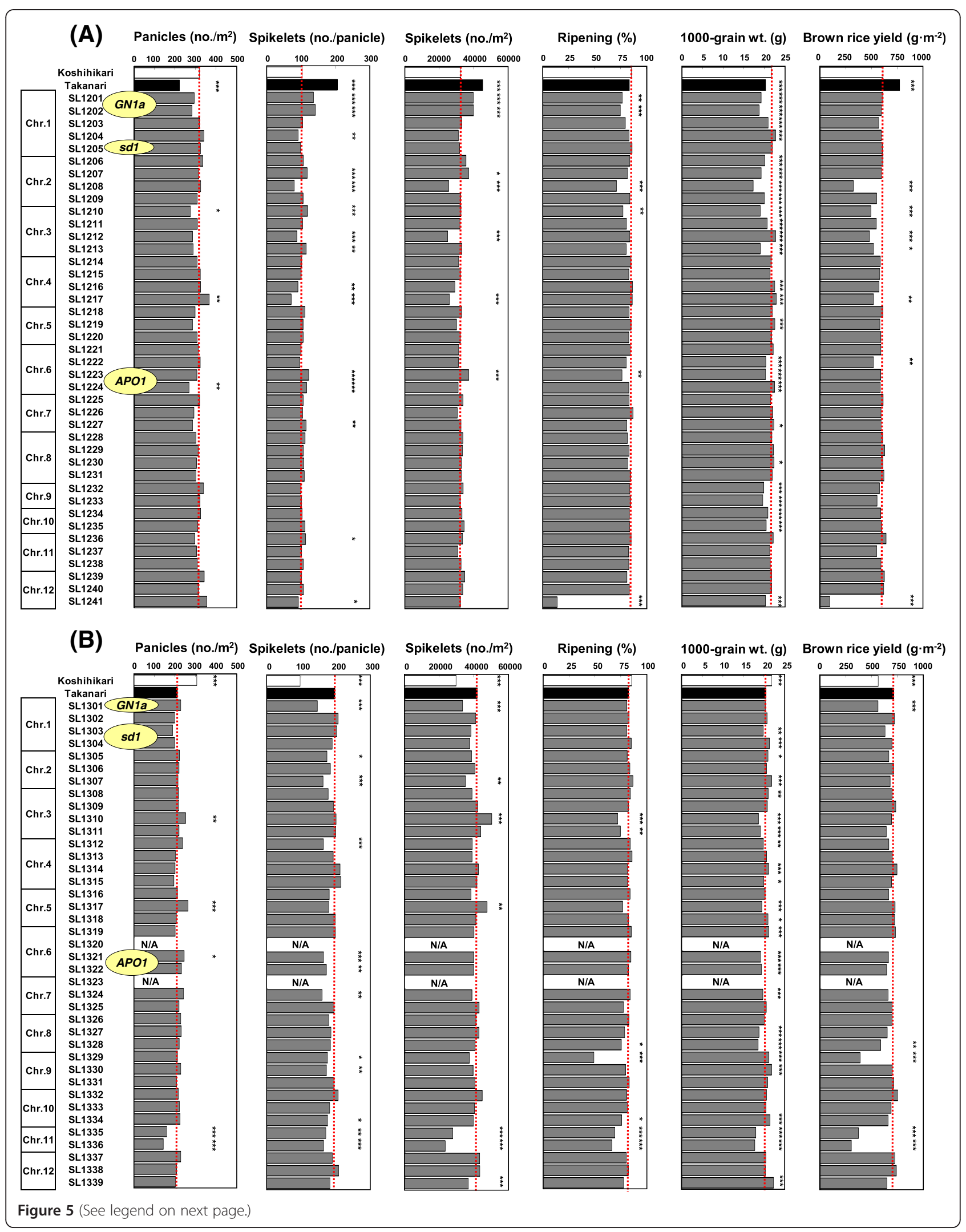


(See figure on previous page.)

Figure 5 Yield and its components for the chromosome segment substitution lines (CSSLs) in the Koshihikari (A) and Takanari (B) backgrounds. Bars indicate mean values over two years. Dashed red lines denote trait values in Koshihikari (A) and Takanari (B). ${ }^{*} * P<0.001$, ${ }^{*} P<0.01$ and ${ }^{*} P<0.05$ versus Koshihikari (A) and Takanari (B), assessed by Dunnett's test. N/A, not available. GN1a, sd1, and APO1 adjacent to the name of a CSSL indicate that the CSSL carries that gene.

In the Takanari background, four QTLs were found for panicle number, three of which increased and one of which decreased panicle number in plants with the Koshihikari allele (Figure 6B). Nine QTLs for number of spikelets per panicle were detected, and all had negative effects in plants with the Koshihikari allele. Considering the effects of the QTLs and the chromosomal regions, the loci on the short arm of chromosome 1 and on the long arm of chromosome 6 were regarded as GN1a and APO1, respectively. Six QTLs were found for number of spikelets per square meter; two increased and four decreased spikelet number in plants with the Koshihikari allele. Five QTLs for ripening percentage had negative effects in plants with the Koshihikari allele. Nineteen QTLs were detected for 1000grain weight; ten increased and nine decreased 1000-grain weight in plants with the Koshihikari allele. Four QTLs for brown rice yield were identified; all had negative effects on yield in plants with the Koshihikari allele.

\section{Discussion}

Rice yield is a highly complex trait and is controlled by a large number of QTLs with small individual effects.

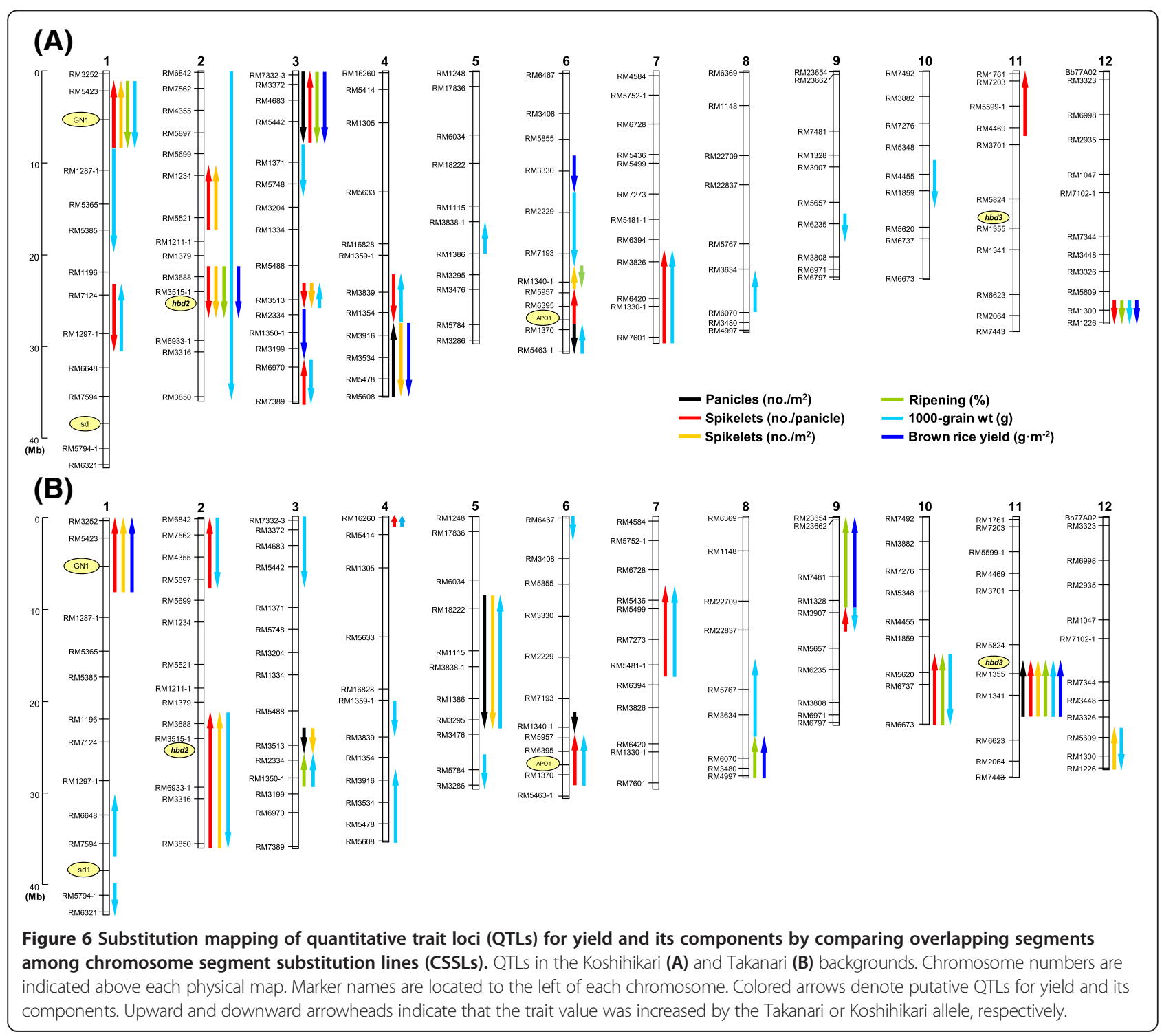


CSSLs are appropriate for detecting QTLs with both large and small effects, and reciprocal CSSLs confer the advantage of enabling evaluation of differences in allelic effects of QTLs in both genetic backgrounds. If a detected QTL shows the same gene activity in reciprocal genetic backgrounds, that locus should have no genetic interaction or epistasis with other background factor(s). QTLs that show different gene activity in the reciprocal backgrounds may be involved in genetic interaction or epistasis with other background factor(s) [21]. By using reciprocal CSSLs derived from a cross between Takanari and Koshihikari, we detected a number of QTLs underlying brown rice yield and its components. Among these loci, we confirmed that the Takanari alleles of GN1 $a$ and APO1 increased the number of spikelets per panicle in the reciprocal backgrounds (Figures 5 and 6). A QTL for the number of spikelets per unit area, at RM3513 on chromosome 3, and QTLs for 1000-grain weight at RM3634 and RM1300 on chromosomes 8 and 12, respectively, exhibited the same gene activity in both genetic backgrounds. These results indicate that these five QTLs should be a single factor, unless multiple genes associated with the trait were located in the substituted segment. Thus, favorable alleles from these QTLs can be used to improve target traits in either of the reciprocal genetic backgrounds. Furthermore, a recent study cloned a QTL on the long arm of chromosome 8 as GW8 controlling grain size [32]. Because the position of the QTL for 1000-grain weight is close to $G W 8$, there is a possibility that the QTL detected in this study is GW8. Further study is necessary to confirm this.

On the other hand, the remaining QTLs were detected in only one of the two genetic backgrounds, suggesting that these loci may be under epistatic control with other gene(s) in the background. A possible epistatic interaction was observed for QTL clusters at RM3515-1 on chromosome 2 in the Koshihikari background and at RM1355 on chromosome 11 in the Takanari background (Figure 6). These QTL clusters were not detected at the same genomic regions in the opposite background. SL1208, carrying the QTL cluster on chromosome 2, and SL1335 and SL1336, which carried the QTL cluster on chromosome 11 , showed hybrid weakness (delayed heading, dwarf plant stature, fewer spikelets, lower ripening percentage, and lower yield) (Figure 5, Additional file 2: Figure S2). A previous study revealed that hybrid breakdown is caused by interaction of two recessive genes, $h b d 2$ and $h b d 3$, and that Koshihikari carries $h b d 3$ and an indica cultivar, Habataki, which is a sister line to Takanari, carries $h b d 2$ [33]. The $h b d 2$ and $h b d 3$ genes are located in the vicinity of QTL clusters on chromosomes 2 and 11, respectively. Assuming that Takanari also carried $h b d 2$, the two QTL clusters and hybrid weakness observed in SL1208, SL1335, and SL1336 can be well explained because SL1208 should have carried $h b d 2$ and $h b d 3$ in the Koshihikari background and SL1335 and SL1336 also carried $h b d 2$ and $h b d 3$ in the Takanari background. Therefore, the two QTL clusters should be a result of interaction between $h b d 2$ and $h b d 3$. Although we could not elucidate gene interactions for other QTLs detected in only one of the reciprocal backgrounds, detection of many QTLs in only one genetic background suggests that a large part of the variation in yield and its components between Takanari and Koshihikari may be controlled by gene interactions. This is the first study to suggest the possibility of epistatic control of yield and its components on the basis of reciprocal CSSLs. Further studies are necessary to identify background factors that interact with the detected QTLs.

Mapping of QTLs also revealed a trade-off among yield components. A notable example was that the allele of the QTL associated with increased sink size was associated with decreased ripening percentage or 1000-grain weight. This trade-off was observed for seven chromosomal regions in the Koshihikari background (including GN1a and APO1) and six in the Takanari background (Figure 6). The trade-off might be caused by a shortage of source strength or carbohydrate translocation, or might be caused by an imbalance among sink size, source strength, and translocation capacity, resulting in no increase in final yield. A lack of remarkable increase in grain yield was also reported for NILs containing the favorable allele of GN1a and APO1 in other japonica genetic backgrounds [34]. However, it should be noted that the parental cultivar, Takanari, obtained a high ripening percentage despite a large sink size derived from the favorable allele of GN1a and APO1. The high ripening percentage in Takanari is considered to be caused by the strong source (high photosynthesis rate) and high carbohydrate translocation capacity [11-14]. These results suggest that QTLs for increased source strength and translocation capacity should be found in the Takanari allele and that the combination of GN1a or APO1 with these QTLs would be necessary to attain higher yield in the Koshihikari background. Recently, a QTL for high leaf photosynthesis was identified in Takanari and cloned as a single gene (GPS) in the same genetic combination between Takanari and Koshihikari [35]. We are currently developing a pyramid line carrying GN1a and GPS to test yield increase in the Koshihikari background.

Although the Takanari allele of GN1a did not contribute to yield increase in the Koshihikari background, the Koshihikari allele of GN1a decreased sink size traits (spikelet number per panicle and per square meter) and thus reduced brown rice yield in the Takanari background (Figure 5B and Figure 6B). These results indicate that the Takanari allele of GN1a is required to 
achieve high yield potential in Takanari. These results and the strong source in Takanari also imply that yield potential in Takanari might be increased by enlarging its sink size. We identified a QTL that increased panicle number and spikelet number per square meter on the long arm of chromosome 3 in the Takanari background (Figure 6B). SL1310, which carried this QTL, produced $20 \%$ more spikelets per square meter than Takanari. This is a promising QTL to increase sink size in Takanari. However, SL1310 did not attain higher brown rice yield than Takanari, because of reduced ripening percentage and 1000-grain weight (Figure 5B). These results indicate that it is necessary, even in the Takanari background, to combine the QTL on chromosome 3 with loci that enhance source strength and translocation capacity to raise yield potential. Although Koshihikari has lower leaf photosynthesis than Takanari, our previous study detected a QTL for increasing leaf photosynthesis with the Koshihikari allele in the Takanari background [35]. We are currently combining the QTL on chromosome 3 with the high-photosynthesis QTL in the Takanari background to test the increase in yield potential in Takanari, as well as attempting to clone both QTLs.

We detected six QTLs for yield in the Koshihikari background and four in the Takanari background, but no QTLs for yield common to both backgrounds were detected, and all QTLs had negative effects on yield. As discussed above, the failure to detect common QTLs might be caused by epistatic control of these QTLs. The negative effects might be due to imbalance among sink size, source strength, and translocation capacity caused by the substitution of a QTL. However, the results presented here are based on trials conducted at a single experimental site with a single fertilization treatment. Because yield is often influenced by environmental conditions, further trials under multiple environmental conditions are necessary to confirm the effects of the QTLs detected in this study.

\section{Conclusion}

We have successfully developed reciprocal CSSLs derived from a cross between rice cultivars Takanari and Koshihikari. Genetic analysis by reciprocal CSSLs confirmed their usefulness and indicated that some QTLs for yield and its components represented a single factor, while others may be controlled by epistatic interactions. Substitution mapping also suggested the need to combine sink-size QTLs with source-strength or translocationcapacity QTLs to increase rice yield in both genetic backgrounds. Our results provide a foundation for developing rice cultivars with higher yield potential in future breeding programs.

\section{Additional files}

\begin{abstract}
Additional file 1: Figure S1. Genotype data for 41 chromosome segment substitution lines (CSSLs) in the Koshihikari genetic background and 39 CSSLs in the Takanari genetic background. Columns show CSSLs and rows show DNA markers. $\mathrm{A}, \mathrm{B}$, and $\mathrm{H}$ indicate homozygous for Koshihikari, homozygous for Takanari, and heterozygous, respectively. Approximate positions ( $\mathrm{Mb}$ ) of each marker are based on the physical map distance in Os-Nipponbare-Reference-IRGSP-1.0 [27]. The sequences of the forward and reverse primers were $5^{\prime}$-CTCCATCCCAAAATAAGTTC- $3^{\prime}$ and 5'-GCTGGCCTGTCATCC-3' for GN1a, 5'-AGCTGGACATGCCCGTGGTC-3' and 5'-TTGAGCTGCTGTCCGCGAAG-3' for sd1, and 5'-CCGGTTTTGGTTTGTCTCAG-3' and 5'-ATGAACACTGTCCAACAAATTGTTT-3' for APO1, respectively.
\end{abstract}

Additional file 2: Figure S2. Days-to-heading and culm length of chromosome segment substitution lines (CSSLs) in the Koshihikari (A) and Takanari (B) backgrounds. Bars indicate mean values over two years. Dashed red lines denote trait values in Koshihikari (A) and Takanari (B). ${ }^{* *} P<0.001$, ${ }^{* *} P<0.01$, and ${ }^{*} P<0.05$ versus Koshihikari (A) and Takanari (B), determined by Dunnett's test. N/A, not available.

Additional file 3: Figure S3. Sequence polymorphisms of GN1a, APO1, and sd 1 between Koshihikari and Takanari. Light blue bars represent exons; white bars represent $5^{\prime}$ and $3^{\prime}$ untranslated regions.

\section{Competing interests}

The authors declare that they have no competing interests.

\section{Authors' contributions}

$\Pi$ and TY designed the study and wrote the manuscript. TT, TI, KK, YN, NO, and TY developed the reciprocal CSSLS. TT, YS-A, SY, and HN performed the field experiments. MK and MY participated in the design and coordination of the study. All authors read and approved the final manuscript.

\section{Acknowledgments}

This work was supported by grants from the Ministry of Agriculture, Forestry and Fisheries of Japan (Genomics for Agricultural Innovation, QTL1002 and NVR-0001 and Genomics-based Technology for Agricultural Improvement, RBS2005).

\section{Author details}

${ }^{1}$ NARO Institute of Crop Science, Tsukuba, Ibaraki 305-8518, Japan. ${ }^{2}$ National Institute of Agrobiological Sciences, Tsukuba, Ibaraki 305-8602, Japan. ${ }^{3}$ Institute of the Society for Techno-innovation of Agriculture, Forestry and Fisheries, Tsukuba, Ibaraki 305-0854, Japan.

Received: 8 July 2014 Accepted: 17 October 2014

Published online: 18 November 2014

\section{References}

1. McCouch S, Baute GJ, Bradeen J, Bramel P, Bretting PK, Buckler E, Burke JM, Charest D, Cloutier S, Cole G, Dempewolf H, Dingkuhn M, Feuillet C, Gepts P, Grattapaglia D, Guarino L, Jackson S, Knapp S, Langridge P, Lawton-Rauh A, Lijua Q, Lusty C, Michael T, Myles S, Naito K, Nelson RL, Pontarollo R, Richards CM, Rieseberg L, Ross-lbarra J, et al: Agriculture: feeding the future. Nature 2013, 499:23-24.

2. Khush GS: What it will take to feed 5.0 billion rice consumers in 2030 . Plant Mol Biol 2005, 59:1-6.

3. Tester $M$, Langridge $P$ : Breeding technologies to increase crop production in a changing world. Science 2010, 327:818-822.

4. Peng S, Cassman KG, Virmani SS, Sheehy J, Khush GS: Yield potential trends of tropical rice since the release of IR8 and the challenge of increasing rice yield potential. Crop Sci 1999, 39:1552-1559.

5. Evenson RE, Gollin D: Assessing the impact of the green revolution, 1960 to 2000. Science 2003, 300:758-762.

6. Peng S, Khush GS, Virk P, Tang Q, Zou Y: Progress in ideotype breeding to increase rice yield potential. Field Crop Res 2008, 108:32-38.

7. Kushibuchi K: Historical changes in rice cultivars. 4. Classification and distribution of rice cultivars in Japan. In Science of the Rice Plant (Vol 3). Edited by Matsuo T, Futsuhara Y, Kikuchi F, Yamaguchi H. Tokyo: Food and Agricultural Policy Research Center; 1997:854-875. 
8. Yoshinaga S, Takai T, Arai-Sanoh Y, Ishimaru T, Kondo M: Varietal differences in sink production and grain-filling ability in recently developed high-yielding rice (Oryza sativa L.) varieties in Japan. Field Crop Res 2013, 150:74-82.

9. Nagata K, Sasaki R, Ohdaira Y, Yoshinaga S: Growth, yield and dry matter production of high-yielding rice in the warmer region of Japan. Jpn J Crop Sci 2009, 78(Extra1):240-241.

10. Takai T, Arai-Sanoh Y, Iwasawa N, Hayashi T, Yoshinaga S, Kondo M: Comparative mapping suggests repeated selection of the same quantitative trait locus for high leaf photosynthesis rate in rice high-yield breeding programs. Crop Sci 2012, 52:2649-2658.

11. Chen CP, Sakai H, Tokida T, Usui Y, Nakamura H, Hasegawa T: Do the rich always become richer? Characterizing the leaf physiological response of the high-yielding rice cultivar Takanari to free-air $\mathrm{CO}_{2}$ enrichment. Plant Cell Physiol 2014, 55:381-391.

12. Ohsumi A, Hamasaki A, Nakagawa H, Yoshida H, Shiraiwa T, Horie T: A model explaining genotypic and ontogenetic variation of leaf photosynthetic rate in rice (Oryza sativa) based on leaf nitrogen content and stomatal conductance. Ann Bot 2007, 99:265-273.

13. Takai T, Matsuura S, Nishio T, Ohsumi A, Shiraiwa T, Horie T: Rice yield potential is closely related to crop growth rate during late reproductive period. Field Crop Res 2006, 96:328-335.

14. Taylaran RD, Adachi S, Ookawa T, Usuda H, Hirasawa T: Hydraulic conductance as well as nitrogen accumulation plays a role in the higher rate of leaf photosynthesis of the most productive variety of rice in Japan. J Exp Bot 2011, 62:4067-4077.

15. Yano M: Genetic and molecular dissection of naturally occurring variation. Curr Opin Plant Biol 2001, 4:130-135.

16. International Rice Genome Sequencing Project: The map-based sequence of the rice genome. Nature $2005,436: 793-800$.

17. Ebitani T, Takeuchi Y, Nonoue Y, Yamamoto T, Takeuchi K, Yano M: Construction and evaluation of chromosome segment substitution lines carrying overlapping chromosome segments of indica rice cultivar 'Kasalath' in a genetic background of japonica elite cultivar 'Koshihikari'. Breed Sci 2005, 55:65-73.

18. Keurentjes JJ, Bentsink L, Alonso-Blanco C, Hanhart CJ, Blankestijn-De Vries $H$, Effgen S, Vreugdenhil D, Koornneef M: Development of a near-isogenic line population of Arabidopsis thaliana and comparison of mapping power with a recombinant inbred line population. Genetics 2007, 175:891-905.

19. Ando T, Yamamoto T, Shimizu T, Ma XF, Shomura A, Takeuchi Y, Lin SY, Yano M: Genetic dissection and pyramiding of quantitative traits for panicle architecture by using chromosomal segment substitution lines in rice. Theor Appl Genet 2008, 116:881-890.

20. Hori K, Sugimoto K, Nonoue Y, Ono N, Matsubara K, Yamanouchi U, Abe A, Takeuchi Y, Yano M: Detection of quantitative trait loci controlling pre-harvest sprouting resistance by using backcrossed populations of japonica rice cultivars. Theor App/ Genet 2010, 120:1547-1557.

21. Kubo T, Aida Y, Nakamura K, Tsunematsu H, Doi K, Yoshimura A: Reciprocal chromosome segment substitution series derived from Japonica and Indica cross of rice (Oryza sativa L.). Breed Sci 2002, 52:319-325.

22. Takai T, Nonoue Y, Yamamoto SI, Yamanouchi U, Matsubara K, Liang ZW, Lin HX, Ono N, Uga Y, Yano M: Development of chromosome segment substitution lines derived from backcross between indica donor rice cultivar 'Nona bokra' and japonica recipient cultivar 'Koshihikari'. Breed Sci 2007, 57:257-261.

23. Xu J, Zhao Q, Du P, Xu C, Wang B, Feng Q, Liu Q, Tang S, Gu M, Han B, Liang $G$ : Developing high throughput genotyped chromosome segment substitution lines based on population whole-genome re-sequencing in rice (Oryza sativa L.). BMC Genomics 2010, 11:656.

24. McCouch SR, Teytelman L, Xu Y, Lobos KB, Clare K, Walton M, Fu B, Maghirang R, Li Z, Xing Y, Zhang Q, Kono I, Yano M, Fjellstrom R, DeClerck G, Schneider D, Cartinhour S, Ware D, Stein L: Development and mapping of 2240 new SSR markers for rice (Oryza sativa L.). DNA Res 2002, 9:199-207.

25. Monna L, Kitazawa N, Yoshino R, Suzuki J, Masuda H, Maehara Y, Tanji M, Sato M, Nasu S, Minobe Y: Positional cloning of rice semidwarfing gene, $s d-1$ : rice "green revolution gene" encodes a mutant enzyme involved in gibberellin synthesis. DNA Res 2002, 9:11-17.

26. Terao T, Nagata K, Morino K, Hirose T: A gene controlling the number of primary rachis branches also controls the vascular bundle formation and hence is responsible to increase the harvest index and grain yield in rice. Theor Appl Genet 2010, 120(5):875-893.

27. Kawahara Y, de la Bastide M, Hamilton JP, Kanamori H, McCombie WR, Ouyang S, Schwartz DC, Tanaka T, Wu J, Zhou S, Childs KL, Davidson RM Lin H, Quesada-Ocampo L, Vaillancourt B, Sakai H, Lee SS, Kim J, Numa H, Itoh T, Buell CR, Matsumoto T: Improvement of the Oryza sativa Nipponbare reference genome using next generation sequence and optical map data. Rice 2013, 6:4.

28. Yano M, Katayose Y, Ashikari M, Yamanouchi U, Monna L, Fuse T, Baba T, Yamamoto K, Umehara Y, Nagamura Y, Sasaki T: Hd1, a major photoperiod sensitivity quantitative trait locus in rice, is closely related to the arabidopsis flowering time gene CONSTANS. Plant Cell 2000, 12:2473-2483.

29. Ashikari M, Sakakibara H, Lin S, Yamamoto T, Takashi T, Nishimura A, Angeles ER, Qian Q, Kitano H, Matsuoka M: Cytokinin oxidase regulates rice grain production. Science 2005, 309:741-745.

30. Ookawa T, Hobo T, Yano M, Murata K, Ando T, Miura H, Asano K, Ochiai Y, Ikeda M, Nishitani R, Ebitani T, Ozaki H, Angeles ER, Hirasawa T, Matsuoka M: New approach for rice improvement using a pleiotropic QTL gene for lodging resistance and yield. Nat Commun 2010, 1:132.

31. Sasaki A, Ashikari M, Ueguchi-Tanaka M, Itoh H, Nishimura A, Swapan D, Ishiyama K, Saito T, Kobayashi M, Khush GS, Kitano H, Matsuoka M: A mutant gibberellin-synthesis gene in rice. Nature 2002, 416:701-702.

32. Wang S, Wu K, Yuan Q, Liu Z, Lin X, Zeng R, Zhu H, Dong G, Qian Q, Zhang G, Fu X: Control of grain size, shapes and quality by OsSPL16 in rice. Nat Genet 2012, 44:950-954.

33. Yamamoto E, Takashi T, Morinaka $Y$, Lin S, Wu J, Matsumoto T, Kitano $H_{4}$ Matsuoka M, Ashikari M: Gain of deleterious function causes an autoimmune response and Bateson-Dobzhansky-Muller incompatibility in rice. Mol Gen Genet 2010, 283:305-315.

34. Ohsumi A, Takai T, Ida M, Yamamoto T, Arai-Sanoh Y, Yano M, Ando T, Kondo M: Evaluation of yield performance in rice near-isogenic lines with increased spikelet number. Field Crop Res 2011, 120:68-75.

35. Takai T, Adachi S, Taguchi-Shiobara F, Sanoh-Arai Y, Iwasawa N, Yoshinaga S, Hirose S, Taniguchi Y, Yamanouchi U, Wu J, Matsumoto T, Sugimoto K, Kondo K, Ikka T, Ando T, Kono I, Ito S, Shomura A, Ookawa T, Hirasawa T, Yano M, Kondo M, Yamamoto T: A natural variant of NAL1, selected in high-yield rice breeding programs, pleiotropically increases photosynthesis rate. Sci Rep 2013, 3:2149.

\section{doi:10.1186/s12870-014-0295-2}

Cite this article as: Takai et al:: Genetic mechanisms underlying yield potential in the rice high-yielding cultivar Takanari, based on reciprocal chromosome segment substitution lines. BMC Plant Biology 2014 14:295.

\section{Submit your next manuscript to BioMed Central and take full advantage of:}

- Convenient online submission

- Thorough peer review

- No space constraints or color figure charges

- Immediate publication on acceptance

- Inclusion in PubMed, CAS, Scopus and Google Scholar

- Research which is freely available for redistribution 